\title{
BMJ Open PLAN-e-PSY, a mobile application to improve case management and patient's functioning in first episode psychosis: protocol for an open-label, multicentre, superiority, randomised controlled trial
}

Frédéric Haesebaert (10 ,1,2 Sofia El Oussoul, ${ }^{1}$ Amélie Pavard, ${ }^{1}$ Delphine Fabre, ${ }^{2,3}$ Caroline Cellard, ${ }^{4}$ Laurent Magaud, ${ }^{5}$ Fabien Subtil,,${ }^{6,7}$ Eleonore Damiolini, ${ }^{5}$ Eric Fakra, 2,8 Julie Haesebaert ${ }^{5,9}$

To cite: Haesebaert $F$, El Oussoul S, Pavard A, et al. PLAN-e-PSY, a mobile application to improve case management and patient's functioning in first episode psychosis: protocol for an open-label, multicentre, superiority, randomised controlled trial. BMJ Open 2021;11:e050433. doi:10.1136/ bmjopen-2021-050433

- Prepublication history and additional supplemental material for this paper are available online. To view these files, please visit the journal online (http://dx.doi.org/10.1136/ bmjopen-2021-050433).

Received 20 February 2021 Accepted 10 August 2021

\section{D) Check for updates}

(c) Author(s) (or their employer(s)) 2021. Re-use permitted under CC BY-NC. No commercial re-use. See rights and permissions. Published by BMJ.

For numbered affiliations see end of article.

\section{Correspondence to} Dr Frédéric Haesebaert; Frederic.HAESEBAERT@ch-levinatier.fr

\section{ABSTRACT}

Introduction The prognosis of first episode psychosis (FEP), which is a severe disorder, can be notably impaired by patients' disengagement from healthcare providers. Coordinated specialty care with case management is now considered as the gold standard in this population, but there are still challenges for engagement with subsequent functional impairments. Youth-friendly and patient-centred clinical approaches are sought to improve engagement in patients with FEP. Mobile applications are widely used by young people, including patients with FEP, and can increase the youth friendliness of clinical tools. We hypothesise that a co-designed mobile application used during case management can improve functioning in patients with FEP as compared with usual case management practices.

Methods and analysis A mobile case management application for planning and monitoring individualised care objectives will be co-designed with patients, caregivers and health professionals in a recoveryoriented approach. The application will be compared with usual case management practices in a multicentre, two-arm and parallel groups clinical trial. Patients will be recruited by specialised FEP teams. Impact on functioning will be assessed using the Personal and Social Performance Scale; the variation between baseline and 12 months in each group (control and active) will be the primary outcome.

Ethics and dissemination This study has been approved by the Inserm Institutional Review Board IRB00003888 (Comité d'évaluation éthique de I'INSERM, reference number 20-647). The results of the study will be published in a peer-reviewed journal and presented at national and international conferences. We will also communicate the results to patients and family representatives' associations. An optimised version of the application will be then disseminated through the French FEP network (Transition Network).

Trial registration number ClinicalTrials.gov: NCT04657380

\section{Strengths and limitations of this study}

- The PLAN-e-PSY Study is the first French clinical trial measuring the impact on functioning of a mobile application to improve case management.

- The application is anchored in a recovery-oriented approach to improve its relevance.

- Co-design methods should improve relevance and efficacy of the application.

- The nature of the intervention does not allow blinding, but the involvement of a blinded assessor reduces the risk of bias.

\section{INTRODUCTION}

\section{Psychosis and early intervention}

First episode psychosis (FEP) affects 3\% of the population-mainly adolescents and young adults, the majority of whom progress to psychotic disorder ${ }^{12}$ - and has severe consequences, such as suicidal behaviour. ${ }^{3}$

From the first full-blown symptoms to the next $2-5$ years, the early stages of psychotic disorders represent an opportunity to targeted care and prevention. It is a critical period $^{4}$ as clinical prognosis is worsened when intervention is delayed and the duration of untreated psychosis is increased,,$^{5}$ and is a key period to reduce mortality as it is characterised by an elevated risk of suicide ${ }^{6}$ and low physical health outcomes. ${ }^{7}$ In addition to the symptomatic components, this period is also critical for personal growth at educational, professional and emotional levels. ${ }^{8}$

A negative course can be avoided by the establishment of 'early intervention in psychosis, ${ }^{9}$ which is constituted by a set of integrated supports that lead to a reduction in the symptoms of the disease, promote recovery, and foster better social and 
professional functioning (for review and meta-analysis see Correll et al $2018^{10}$ ).

\section{Case management: a strategy to promote recovery in early psychosis}

Early intervention programmes involve multidisciplinary teams, including a care coordination function embodied by a 'case manager'. Case management requires essential qualities/skills such as: accessibility, flexibility, optimism and competence. ${ }^{11}$ The missions of case managers include assessing the patient's needs, developing a care plan to meet the latter, organising access to the different components of the care plan, monitoring and evaluating care, and providing clinical follow-up. ${ }^{12}$ These missions are broken down into several actions and the content is adapted to the duration of care according to the severity of the disorder and the level of remission. This model of intervention is effective for patients with FEP and is now considered as a 'standard' of care for this population. ${ }^{13}$

\section{Psychosis and engagement in healthcare: a critical situation}

'Patient engagement' refers to the process of building the capacity of patients to facilitate and support their active involvement in their own care. In psychotic disorders, the engagement in the care process is fragile, particularly in the context of FEP that is associated with a high risk of care disengagement, often leading to a relapse; between $20 \%$ and $40 \%$ of patients with FEP disengage from care despite significant therapeutic needs, ${ }^{14}$ and the relapse rate 1 year after stopping treatment ranges from $28 \%{ }^{15}$ to $67 \%$ after a FEP. ${ }^{16}$ Though FEP services exhibit better engagement rates when compared with usual care, it still remains a critical issue, notably due to the variety and complexity of its determinants in patients with FEP. ${ }^{17}$ Several factors at the individual level can influence engagement (eg, substance abuse, trauma history, severity of psychotic or general psychopathology, poor premorbid social adjustment) but determinants at the service level can also interfere with engagement. Among these, one of the most important is the perception that care delivered is not adapted to the patients' needs. ${ }^{18}$ Contrary to other countries in Europe, in France the implementation of FEP services is still at the start and important delays in their implementation had been pointed out recently. ${ }^{19}$ Despite national efforts to bridge the gap between knowledge and clinical routine, until recent years, initiatives were limited to expert centres in university hospitals. ${ }^{20}$ More recently FEP services disseminate in different French regions. ${ }^{21}$ In this context there is an urgent need to translate evidence-based data into clinical tools tailored for the FEP services context. ${ }^{19}$ It this vein, it is essential that case managers involved in FEP services have access to tools designed according to the patient needs and not solely to symptoms, in a recovery-oriented and biopsychosocial approach, to foster the feeling of commitment of patients in their care process.
Mobile applications in mental health: a promising way to promote engagement in FEP services

The use of mobile applications for smartphones represents an interesting perspective to improve the engagement of patients with FEP in care. ${ }^{22}$ Mobile applications now appear as promising tools for better engagement in care, and for better self-management capabilities and better coordination of resources. ${ }^{23}$ Recent data show an increase in the rate of smartphone equipment in the population of patients with psychosis (over 80\%) and qualitative data in this population show that this type of tool is in high demand, in particular to promote the link between the patient and the services and to strengthen the self-management capacities. ${ }^{24}$

Despite the fact that some applications specifically designed for patients with FEP exist and have shown promising results, ${ }^{25}$ none have been developed in French language and evaluated in the French healthcare system. Anchoring the design of the application in a recovery theory would allow to better align the actions resulting from the use of the application with the patient's needs. Moreover, it has recently been shown that developing tools using a 'User-centred design' methodology is feasible in populations with FEP. ${ }^{26}$ We hypothesise that this method is particularly suitable for a 'recovery-oriented' approach.

\section{Research hypothesis}

Our hypothesis is that the use of a mobile case management application for planning and monitoring individualised care objectives, co-designed with patients, their carers and health professionals, improves the functioning of patients managed for FEP, compared with usual case management.

\section{Objectives}

Main objective

The main objective is to evaluate, in a randomised controlled trial (RCT), the efficacy at 12 months of the use of a co-designed recovery-centred planning mobile application (PLAN-e-PSY planning app) in case management to improve the functioning of patients managed for an FEP, compared with usual case management practices within the same services.

\section{Secondary objectives}

The secondary objectives of this study are:

A. Patient-related objectives

1. To study the impact of the PLAN-e-PSY planning application at 6 months, compared with usual practice, on the change of patient functioning,

2. To study the impact of the 12-month PLAN-e-PSY planning application compared with usual practice on days of hospitalisation for psychosis relapse.

3. To study the impact of using the PLAN-e-PSY planning application at 6 months and 12 months compared with usual practice on:

a. The change of engagement in patient care, 
b. The change of the therapeutic alliance between the patient and the case manager,

c. The change of the patient's adherence to pharmacological treatments,

d. The change of the patient's psychotic symptomatology,

e. The change of the patient's level of recovery,

f. The change of the patient's quality of life,

g. The change of the patient's level of activation (empowerment),

h. Patient satisfaction with his or her case management follow-up.

B. Implementation process

To study the implementation of the PLAN-e-PSY application in case management in the intervention group at 12 months.

\section{METHODS AND ANALYSIS}

\section{Type of study}

This is a prospective multicentre open label RCT comparing two parallel open arms. A mixed method will be used, associating to the RCT a qualitative evaluation through semistructured interviews. Randomisation will be stratified on the case manager. There is no blinding for the patient or the case manager, but evaluation will be carried out by a blinded psychiatrist.

The study will be conducted at four centres (with already existing clinical and teaching collaboration) in two regions of France.

This study will be carried out in two successive phases:

1. Co-design phase of the intervention based on the iterative 'user-centred design' approach, involving representatives of carers, patients and caregivers to define the content and form of the smartphone application during co-building workshops. For practical reasons, the workshops phase will take place in Lyon which is the leading site with the involvement of patients from two participating centres (Lyon and Saint-Etienne sites).

2. Deployment and evaluation of the effect of the intervention in an RCT, including mixed quantitative and qualitative approaches, and a study of the implementation of the intervention.

\section{Phase 1: User-centred design co-building process}

The co-design of the intervention takes place in two major successive stages: (see figure 1) (1) Co-design workshops with user committees to obtain version 1, then (2) Testing on a sample of patients (some of whom will have participated in the workshops) to obtain version 2 which will be evaluated during the RCT.

\section{Co-design workshops of user committees to obtain version 1 The 'recovery' approach, as the conceptual framework of the workshops}

In order to improve the overall impact of the care process in patients and their functioning, the application will be built in a 'recovery' perspective, in order to better suit patient needs. ${ }^{27}$

Recovery takes place in several dimensions (clinical, functional, social, physical and existential) and considers the person as a whole. ${ }^{28}$ The dimensions of recovery and illustrations are provided in table $1 .^{28} 29$

\section{Organisation of the workshops}

The objectives of the workshop phase is the design and prototyping of version 1 of the application. The method

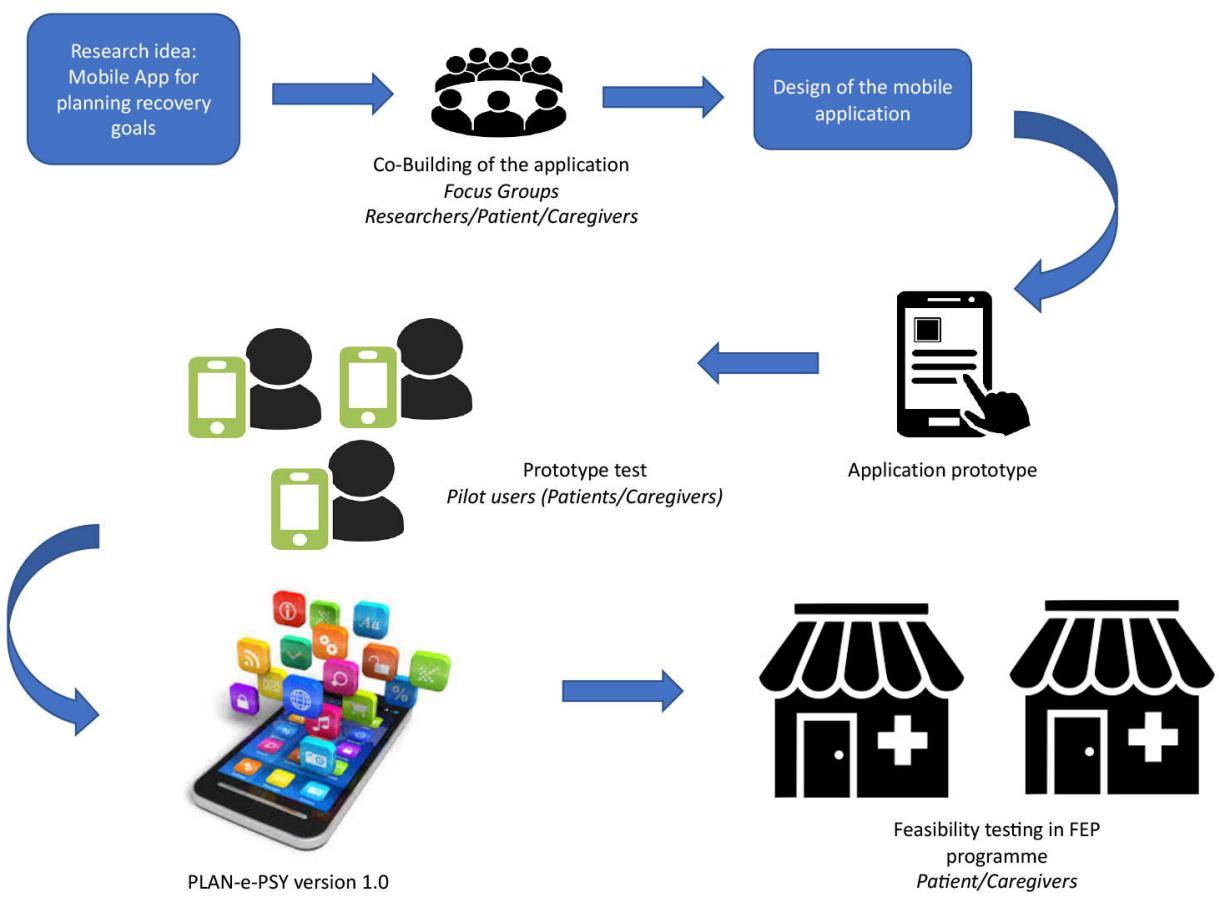

Figure 1 Design diagram of the PLAN-e-PSY application. 


\begin{tabular}{ll} 
Table 1 Dimensions of recovery and their illustrations \\
\hline $\begin{array}{l}\text { Dimensions } \\
\text { of recovery }\end{array}$ & Indicator illustration \\
\hline Clinical & Severity of symptomatology \\
& Number of psychiatric hospitalisations \\
& therapeutic interventions \\
Functional & Accessing and maintaining an employment \\
& in a competitive environment \\
& Access to satisfactory independent \\
& accommodation \\
Social & Network and social support \\
& Diversity and frequency of social activities \\
& Integration into the community, sense of \\
& belonging to a community \\
& Citizenship \\
& Physical health \\
& Weight regulation \\
& Absence of problematic substance or food \\
& consumption \\
Physics & Eeeling of empowerment \\
& Emotional, spiritual well-being \\
Existential & Sense of self-efficacy, autonomy \\
&
\end{tabular}

used is a participatory approach combining co-building workshops and individual interviews in a 'user-centreddesign' approach. This process comprises four main stages: (1) Specification of user needs, (2) Co-building of the application according to user requirements and needs, (3) Evaluation of the tool by the users, (4) Adjustment of the application design according to user feedback. ${ }^{30}$

\section{Patient and public involvement}

The objectives of the PLAN-e-PSY application will be the result of co-building work by balanced joint committees composed of 8 to 10 people. The groups will be composed of:

- Two to six representatives of the target users, that is, patients treated in an FEP service as well as case managers of these same services. Patients will be in a stable phase of their illness and will meet a clinical psychologist involved in the project (AP) to ensure their capacity to actively participate before the inclusion. They will also give their informed consent for the participation in this phase (see online supplemental file).

- A representative of the patients' carers recruited from family associations.

- An associate psychologist, trained in social representations analysis, who will be responsible for observing and collating the exchanges.

- Two facilitators, a psychiatrist with a strong experience in group animation and a peer helper will present the objectives of each session and answer questions and comments from the participants. The psychiatrist ensures that the group runs smoothly and that there is a balance in the distribution of speaking time and good group dynamics among the stakeholders.

- The developer of the application who will be able to join the committee in order to discuss in real time the feasibility of the proposals that are made. The developer may also prepare a first version of the application in the form of a sketch, with a view to materialise the exchanges and provide the committee with an illustrated design of the application.

The aim of these meetings will be to collaboratively build the prototype of the patient application which will be used with the case manager to define the care objectives and ensure their follow-up.

Four meetings will be organised over a period of 2 months. The quality of the workshop process will be improved by postsession debriefing with the facilitators (psychiatrist and peer helper), the psychologist and the steering comity. A balanced involvement of all stakeholders will be a priority for the committee, and adjustments may be proposed before each workshop.

\section{Feasibility testing}

Following the design of the first version of the application by the developer, in conjunction with the co-building committee, an initial test will be carried out on a sample of a minimum of five patients in the four FEP services until data saturation. After this step, an improved version of the application will be used and evaluated in the study presented here.

\section{Phase 2: RCT}

Intervention studied: PLAN-e-PSY mobile application

The version of the application to be evaluated here will result from the process described above; therefore, the full version cannot be presented here. It will be an optimised version of the prototype described below.

\section{Minimum specifications of the application prototype}

The main purpose of the application will be to assist in the definition of patients' goals using an approach rooted in recovery theory, with the monitoring of goal achievement in several stages. The formulation of objectives will thus be done in several stages (illustrated in figure 2 through an example of a possible prototype):

- A first stage of discussion with the patient and the case manager: The case manager introduces the five dimensions (clinical, existential, functional, physical and social) by citing a typical example for each dimension. He or she then asks the patient to list the points that could be improved in each dimension.

- In a second step, the patient selects up to five specific objectives per dimension in total during a discussion with the case manager, assisted by the application. The app requires to position the level of achievement of each objective on a Likert Scale: from 'not at all achieved' to 'achieved'.

- In a third step, the patient, accompanied by the case manager, will have to list the actions to be carried 


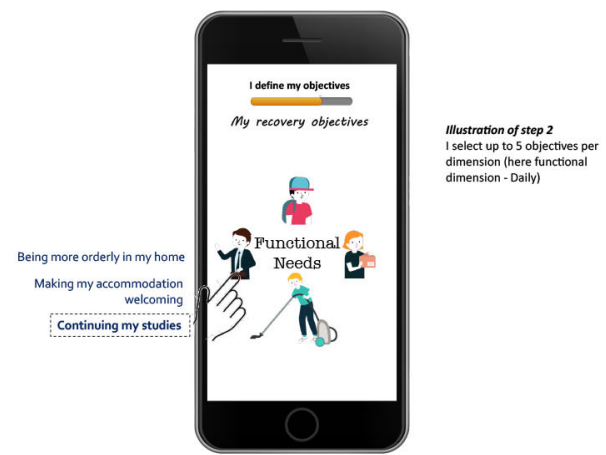

Figure 2 Example of a possible prototype for PLAN-e-PSY.

out to achieve his or her objectives. Each action could require the intervention of a facilitator and/ or the use of a psychoeducational information sheet or an online resource that can help the user in their recovery process. The whole process of defining objectives should not exceed 15 days.

An evaluation of the achievement of the objectives will then be requested weekly by the application through notifications. These will serve as a reminder so that the patient can carry out a regular evaluation and update of his or her project. If the patient reaches a plateau in the achievement of the objective, he or she will receive a positive feedback and the means of increasing the level will be re-examined with the case manager. The objectives will be reviewed regularly with the case manager and systematically every 2 weeks.

The application will have customisable profile management with a nickname and a picture. Warnings signs and a crisis plan can also be added. The app will include a general support area with links to websites with recovery resources. The app will be available on iOS and Android platforms. The development will use technology that allows a similar rendering on both platforms to limit the bias of the type of smartphone used. Since privacy has been identified as one of the priorities of patients in their expectations regarding mobile health applications, ${ }^{31}$ the data will be stored only locally on the phone without transfer to any third-party services.

\section{Comparison-control group}

The FEP services participating in the study offer their patients case management that will correspond to usual treatment of the control group. All the centres are part of the National Transition Network (https://idpsy.org/ reseau-transition/centres/). Care delivery is based on intensive treatment during the critical period of psychosis, drawing on a wide network of caregivers (including the case manager) and non-caregiving professionals, working together towards the successful recovery of the patient. ${ }^{32}$

\section{Randomisation}

Randomisation will be carried out centrally by Interactive Web Response Service (IWRS, ENNOV Clinical solution) using a random list generated by the permuted-block method, and stratified on the case manager to produce balanced 1:1 groups. The allocation sequence will be generated by the statistician in charge of the study, blinded to the coordinating centre and investigators. The randomisation will be performed by the case manager who will connect to the eCRF (ENNOV Clinical randomisation module). The randomisation will allow transmission of individual login connection codes to the application for patients allocated to the intervention group, thus ensuring that patients in the control group cannot download and use the application.

There is no blinding for the patient or the case manager. Nevertheless, the evaluation of the comparative outcomes between the two groups will be carried out by a psychiatrist who is not involved in the follow-up of the patient and who will be blinded to the patient's group.

\section{Qualitative aspects}

This protocol is based on a recovery-centred approach and allows substantial qualitative analysis of the data. In the first phase (co-building process), the user committee representations about recovery and resources will be analysed by a social psychologist and collected to build the first version of the mobile app. This version will be then enhanced by the first users' suggestions.

In the second phase (RCT), semistructured interviews will be conducted in a sample of 15-20 patients from the intervention group (selection based on a voluntary and maximum variation approach regarding centre, age, gender) and with case managers of each participating centre (eight case managers involved in the study) 12 months after the intervention.

Interviews will be conducted until saturation. Face-toface or video interviews will be conducted by a psychologist trained in qualitative research, based on an interview grid design to explore: (1) The user experience, including acceptability, appropriation and use of the application, (2) The perceived benefits and shortcomings of the application, (3) The changes induced by the application in the patient-case manager relationship, and (4) The impact on recovery, care pathway and care adherence.

All interviews will be audio-recorded and transcribed verbatim. Thematic content analysis will be performed using the NVivo software (NVivo QSR international). Triangulation will be conducted by combining the results 
of the patients and the case managers, and by combining the interviews and the quantitative results. The qualitative results will inform the interpretation of the quantitative results on the impact and use of the application.

\section{Outcomes}

\section{Primary outcome}

The main assessment criterion is the variation in the patient's functional score measured by the Personal and Social Performance (PSP) Scale ${ }^{33}$ between inclusion and 12 months, provided by a psychiatrist, trained in the use of the scale, not directly involved in the patient's follow-up and blinded to the patient's allocation group.

PSP is a hetero-evaluative scale used internationally but is also available in French, ensuring operation in a single score that takes into account four domains: productive social activities (work/studies), social network, personal care, and disruptive or aggressive behaviour. The integrative score ranges from 0 to 100 , the higher scores reflecting better functioning. Functioning corresponds to an individual's capacity to assume his or her social role, in the domestic, professional or school, emotional, family and friendship spheres. This is the final objective of casemanaged FEPs.

In terms of psychometric properties, this scale has: ${ }^{33}$

- A Cohen's kappa score weighted at 0.95 , revealing a strong interjudge agreement.

- An intraclass correlation coefficient of 0.98 , reflecting high inter-rater reliability.

\section{Secondary outcomes}

The secondary outcomes, linked to the secondary objectives detailed above, are as follows:

\section{Patient-related outcomes}

1. Change of the functioning score measured by the PSP between inclusion and 6 months in the two groups.

2. Patient evaluation at 12 months in both groups of the number of days of hospitalisation for relapse of psychosis over 12 months, collected at follow-up.

3. Evolution of the following criteria between 0 and 6 months and between 0 and 12 months in both groups:

a. Patient engagement in care as measured by the Service Engagement Scale (SES) translated into French ( Tait $^{34}$ 2002).

b. Therapeutic alliance as measured by the Working Alliance Inventory (WAI), patient scale and caregiver scale (case manager). ${ }^{35}$

c. Adherence to drug treatment as measured by the Medical Adherence Rating Scale (MARS) Score. ${ }^{36}$

d. Psychotic symptomatology assessed by the Positive and Negative Syndrome Scale (PANSS) This heteroquestionnaire gives three scores, positive symptoms, negative symptoms and general psychopathology. The reliability estimated by Cohen's Alpha Index for the positive scale $\alpha \mathrm{p}=0.73$ and negative $\alpha \mathrm{n}=0.83$. The overall reliability is $\alpha=0.79 .^{37}$ e. Recovery measured by the Stages of Recovery Instrument (STORI) $\alpha=0.88-0.94 .^{38}$

f. Quality of life using theSchizophrenia Quality of Life Questionnaire Short Form - 18 items (S-QoL 18) $\alpha=0.72-0.84 .^{39}$

g. Empowerment level measured by the selfadministered Patient Activation Measure (PAM) Questionnaire (Hibbard et al and Hudon et al).

h. Patient satisfaction with case management, evaluated by a self-administered questionnaire using a Visual Analogue Scale.

\section{Implementation process}

Evaluation will be performed at 12 months in the intervention group of the fidelity of use and effective use of the PLAN-e-PSY application by the case manager and the patients, which encompasses: fidelity of use of the defined procedures, use of the different parts of the application, proportion of case management consultations during which the application was not used at 12 months, proportion of patients who stopped using the application at 12 months and reasons given, effective time of use of the application-these elements will be collected from the internal monitoring data of the application.

The acceptability and appropriation of the application by patients and the case manager will be assessed during the semistructured interviews.

\section{Eligibility criteria}

The study will involve patients presenting an FEP, followed in FEP services (four centres in Auvergne-Rhône-Alpes and Bourgogne), and having initiated antipsychotic treatment less than 6 months previously (admission criteria in most FEP services). Participation in the study will be offered to all eligible patients treated in the abovementioned FEP services.

\section{Inclusion criteria}

- Men and women aged between 18 years and 30 years enrolled in a specialised FEP service for a first psychotic episode, defined as follows:

- Presence of daily psychotic symptoms for more than a week that have been characterised at a clinical examination by a psychiatrist.

- Initiation of antipsychotic treatment for less than 6 months.

- A diagnosis of schizophrenia, schizoaffective disorder, schizophreniform disorder or brief psychotic disorder established during the past 6 months according to the Diagnostic and Statistical Manual of Mental Disorders, fifth edition (DSM-5) criteria.

- Mastery of the French language (read and spoken).

- Owning a smartphone.

- Having provided written informed consent.

Criteria for non-inclusion

- Patients in psychiatric intensive care units because of severe agitation/disorganisation.

- Patients under guardianship. 


\section{Premature exit criteria}

As part of the study, every effort will be made to follow-up all patients for the duration of the protocol and to collect the 12-month end point. If necessary, the reasons for withdrawal from the study will be collected: withdrawal of consent, refusal of follow-up, relocation, death.

If the patient is no longer being followed-up in the study without any cause being identified, he or she will be considered lost to follow-up.

\section{Study schedule}

Inclusion will take place following the referral of patients to the participating FEP services. This, as well as the follow-up visits will be carried out by a psychiatrist not directly involved in the patient's follow-up.
Follow-up visits will be carried out respectively at 6 months and 12 months \pm 15 days from the date of inclusion. They will include a clinical examination with the use of different scales that make up the main and secondary objectives of the study (see table 2).

\section{Statistical aspects}

Number of subjects required

The main assessment criterion is the variation in the PSP Score between 0 and 12 months. According to the study reported by Chang et al, ${ }^{40}$ comparing a 'standard' and an 'extended' early intervention programme in a specialist team, the SD of the PSP score is close to 13 at 0 and 12 months in both groups. Assuming a correlation of 0.5 between the PSP scores at 0 and 12 months,

Table 2 Patient follow-up and assessment schedule

\begin{tabular}{|c|c|c|c|c|c|}
\hline Steps & Preinclusion & Inclusion & $\begin{array}{l}\text { Follow-up to } 6 \\
\text { months }\end{array}$ & $\begin{array}{l}\text { Follow-up to } \\
12 \text { months }\end{array}$ & End of study \\
\hline Moment & D-30 to D-15 & Do & M6 \pm 15 days & M12 \pm 15 days & M12 \pm 15 days \\
\hline \multicolumn{6}{|l|}{ Steps } \\
\hline Patient information & $\mathrm{x}$ & & & & \\
\hline Clinical examination & & $x$ & $x$ & $x$ & $x$ \\
\hline Collection of informed consent & & $x$ & & & \\
\hline Verification of inclusion criteria & $\mathrm{x}$ & $x$ & & & \\
\hline Psychiatric history & $x$ & & & & \\
\hline Written informed consent & & $x$ & & & \\
\hline Randomisation & & $x$ & & & \\
\hline Use of the application & & $x$ & $x$ & $x$ & $x$ \\
\hline Evaluation of efficacy & & $x$ & $x$ & $x$ & \\
\hline PSP Scale & & $x$ & $x$ & $x$ & \\
\hline Number of days in hospital & & & $x$ & $x$ & \\
\hline SES & & $x$ & $x$ & $x$ & \\
\hline WAI & & $x$ & $x$ & $x$ & \\
\hline MARS & & $x$ & $x$ & $x$ & \\
\hline PANSS & & $x$ & $x$ & $x$ & \\
\hline STORI & & $x$ & $x$ & $x$ & \\
\hline S-QoL 18 & & $x$ & $x$ & $x$ & \\
\hline PAM (empowerment) & & $x$ & $x$ & $x$ & \\
\hline Satisfaction (VAS) & & $x$ & $x$ & $x$ & \\
\hline Search for adverse events & & $x$ & $\mathrm{x}$ & $\mathrm{x}$ & $x$ \\
\hline
\end{tabular}

Evaluation of implementation in practice

\begin{tabular}{|c|c|c|c|c|c|}
\hline Acceptability & $x$ & $x$ & $x$ & $x$ & \\
\hline Reliability & $x$ & $x$ & $x$ & $x$ & \\
\hline Use in practice & $x$ & $x$ & $x$ & $x$ & \\
\hline
\end{tabular}

MARS, Medication Adherence Rating Scale; PAM, Patient Activation Measure; PANSS, Positive and Negative Syndrome Scale; PSP, Personal and Social Performance; SES, Service Engagement Scale; S-QoL 18, Schizophrenia Quality of Life Questionnaire Short Form; STORI, Stages of Recovery Instrument; VAS, Visual Analogue Scale; WAI, Working Alliance Inventory. 
the SD of the variation in the PSP score would also be 13.

Based on the results of the study reported by Chang et $a l,{ }^{40}$ a 6 -point difference in PSP score variation between the two groups can be expected. Under these hypotheses, the inclusion of 75 subjects per group will lead to a power of $80 \%$ to demonstrate a statistically significant difference between the two groups, for a two-sided alpha level of $5 \%$ (Fleiss et al). ${ }^{41}$

Taking into account $10 \%$ of patients lost to follow-up, it will be necessary to include 84 subjects per group, that is, a total of 168 individuals.

\section{Statistical methods}

\section{General aspects}

A significance threshold of $5 \%$ will be considered in the analyses. The analyses will be carried out using SAS software, V.9.4 (SAS Institute, Cary, North Carolina, USA) and R software. ${ }^{42}$ The analysis of the primary and secondary outcomes will be carried out on the intentionto-treat (ITT) population. Missing data will not be replaced. The primary analysis of the primary end point will be performed using a mixed model assuming that missing data are randomly missing; this will allow to consider all patients for the ITT analysis. A secondary analysis will be performed by replacing the missing score changes with the highest negative changes observed in the study.

\section{Primary outcome}

The change in the PSP Score between inclusion and 12 months will be compared between the two groups using a linear mixed-effects model.

This model will take into account as fixed effect the assessment time (inclusion, 6 months and 12 months; this variable will be taken into account in a qualitative way, or a quantitative one if the PSP evolution over time is linear), the centre, the intervention group and an interaction between the assessment time and the group. This interaction will allow the assessment of the benefit of using the application.

The model will take into account, in random effects, one random intercept per case manager and one random intercept per subject nested at the case manager level, and, depending on the data and on the linearity of the PSP evolution over time, a random slope per case manager, and a random slope per subject nested in the case manager.

A single mixed model will be built that will include the PSP Scores over all three assessment periods.

For the main objective, the effect of application between inclusion and 12 months will be tested only on the basis of the interaction coefficient associated with the group and the 12-month assessment time.

In the second step, the effect of the intervention will be tested at 6 months using the associated interaction coefficient.

\section{Secondary outcomes}

The number of days of rehospitalisation for relapse of psychosis will be compared between the two groups using a Poisson regression model which takes into account as explanatory variable the centre and the group.

Criteria related to the therapeutic alliance between patient and case manager, patient adherence to pharmacological treatments, psychotic symptomatology, level of recovery, quality of life, level of patient activation and commitment to care will be analysed in a similar way to that of the primary outcome.

Patient satisfaction and case management follow-up will be analysed using a linear mixed model taking into account as fixed effect the evaluation time (6 months or 12 months; qualitative variable), the centre and the group, and will include a random intercept per case manager and a random intercept per subject nested at the case manager level.

\section{DISCUSSION}

\section{Strengths and limitations}

The PLAN-e-PSY Study is an open-label, multicentre, superiority, RCT study. The study will take place in FEP services in four psychiatric hospitals (Lyon, Saint-Etienne, Clermont-Ferrand and Dijon).

The study conducted will provide results with a high level of evidence. In addition, we will integrate mixed quantitative and qualitative approaches aimed at obtaining a global and in-depth evaluation of the effects of the intervention on patients and professionals. Finally, we will assess the acceptability of the intervention and its integration into practice so that we can then facilitate its wider deployment and its sustainability after the study is completed.

The primary outcome chosen, improvement in patient functioning, is currently a priority objective of psychiatric care for FEP. ${ }^{13}$ Functioning is associated with quality of life $^{43}$ and is strongly bound to patients' objectives on a recovery-oriented perspective. Patient functioning will be assessed using the PSP. ${ }^{33}$ This is a validated scale in patients within the schizophrenia spectrum, translated into French ${ }^{44}$ and widely used in the literature in diverse settings including in FEP services. ${ }^{45-48}$ It provides an integrative score with good psychometric properties. The choice to consider the 12-month measurement as the main criterion makes it possible to measure the effect of the intervention over a period conditioning the commitment to care and the long-term prognosis.

Secondary end points reflect the different dimensions that may be impacted by the intervention and are potentially predictive of the long-term prognosis. They include several dimensions: clinical and symptomatic (PANSS), progress through the stages of the recovery process (STORI) and quality of life (S-QoL 18). In addition, we will measure commitment to care according to different approaches: the degree of activation or empowerment 
(PAM), engagement in FEP service (SES), therapeutic alliance (WAI) and drug compliance (MARS).

Although the SES is validated in FEP services, most of the measurements for secondary outcomes are tools validated for patients with schizophrenia or along the schizophrenia spectrum, or even, more generally in patients with mental health disorders. Nevertheless, we think these measurements will provide useful information for the future development of tools (digital or not) dedicated to improving case management.

This study cannot be blinded due to the nature of the intervention, involving the use of a mobile application in the intervention group and the usual case management practices in the control group. The intervention requires the active participation of the patient as well as the caregiver. Nevertheless, several elements reduce the risk of potential measurement bias associated with conducting the trial in an open trial: (1) The use of a validated standardised scale (PSP) as the primary outcome, and (2) The collection of this scale by a trained assessor, not involved in the clinical follow-up of the patient, and unaware of the group to which the patient is allocated.

Finally, the feasibility will be increased by an already existing collaboration of the four centres in the clinical and teaching fields. All the centres are part of the National Transition Network (https://idpsy.org/reseautransition/centres/) which will also increase engagement of stakeholders in the research process and feasibility.

\section{Originality and potential benefits of the application}

The originality of our project concerns, on the one hand, the intervention-a mobile monitoring application that will be used jointly by patients and case managers. Smartphone use rates among people with psychotic disorders are close to the general population ${ }^{24}$ and the use of smartphones could increase the use and acceptability of a monitoring tool in the young FEP population. Moreover, the relevance of the application will be reinforced by its design in a recovery-oriented conceptual framework, its adaptation to each stage of management, to the literacy level of patients and to their environment.

On the other hand, the methodological innovation lies on the collaborative and patient-centred design of the application ('user-centred design' approach). These approaches are still underdeveloped in mental health, although aligning care with patients' and carers priorities in a collaborative manner could increase the practice of health professionals, improve the relevance of the care objectives, and promote adherence and motivation. The tool will also make it possible to formulate a personalised care project, individually adapted to each person. According to our hypotheses we expect several benefits of this approach for patients, in terms of functioning; benefits in term of challenging issues in FEP services such as communication with case managers, patients' engagement and treatment adherence, can also be expected.

\section{ETHICS AND DISSEMINATION}

The protocol received ethical approval by the 'Comité d'évaluation éthique de l'INSERM' Institutional Review Board IRB00003888 (reference number 20-647). The final application used for the RCT will be resubmitted for final approval.

The paper refers to version 1 of the protocol (22 September 2019). Important protocol modifications will be registered in the ClinicalTrial.gov registry and communicated to the ethics committee.

The results of the study will be published in a peerreviewed journal. They will also be disseminated through local, national and international conferences. We will also communicate the results to patients and family representatives' associations through partnership meetings. Authorship in the final paper will meet the International Committee of Medical Journal Editor authorship criteria. No professional writers will contribute to the study.

Once published, the application will be optimised and disseminated to the National Transition Network ((https://idpsy.org/reseau-transition/centres/) in French FEP services.

\section{Study committees}

The study steering committee is responsible for overseeing the supervising the implementation and conduct of the study, possible revisions of the protocol, writing reports and publications.

Members of this committee include the principal investigator $(\mathrm{FH})$, the methodologist $(\mathrm{JH})$, the biostatistician (FS), one research professional (LM), one psychologist (ED) and one psychiatrist (EF).

No data monitoring committee was required due to the type of the intervention.

\section{Consent to participate}

Psychiatrists will obtain informed consent from participants, with forms approved by the ethics committee, for each study phase (see online supplemental file 1 for the phase 1 consent form).

\section{Confidentiality}

Personal data will be collected on paper and on electronic data storage and pseudonymised. Only pseudonymised data will be used for the study. Only the investigators and members of the steering committee of the study will have access to the final data set.

\section{Author affiliations}

${ }^{1}$ SUR-CL3R-PEPS, CH Vinatier, Bron, Rhône-Alpes, France

${ }^{2}$ PSYR2 Team, INSERM U1028, CNRS UMR5292, Lyon Neuroscience Research

Center, Université Claude Bernard Lyon 1, Lyon, France, Lyon, France

${ }^{3}$ Service Universitaire des Pathologies Psychiatriques Résistantes, CH Vinatier, Bron, Rhône-Alpes, France

${ }^{4}$ Ecole de Psychologie, Université Laval, Quebec, Québec, Canada

${ }^{5}$ Service Recherche et Epidémiologie Cliniques, Hospices Civils de Lyon, Pôle de Santé Publique, Lyon, Auvergne-Rhône-Alpes, France

${ }^{6}$ Service de biostatistiques, Hospices Civils de Lyon, Pôle de Santé Publique, Lyon, Auvergne-Rhône-Alpes, France 
${ }^{7}$ Laboratoire de Biométrie et Biologie Evolutive, UMR5558, Villeurbanne, RhôneAlpes, France

${ }^{8}$ Pôle Psychiatrie, Centre Hospitalier Universitaire de Saint-Etienne, Saint-Etienne, Rhône-Alpes, France

${ }^{9}$ Université Claude Bernard Lyon 1, Research on Healthcare Performance (RESHAPE), INSERM U1290, Lyon, France

Contributors FH, JH and EF conceptualised the study. SEO, AP, DF, CC, LM, ED gave critical inputs on the co-building process and the design of the protocol. FS designed the statistical analysis plan. FH and SEO wrote the original protocol. FH wrote the manuscript. All authors gave critical inputs to the manuscript and approved the final version.

Funding The PLAN-e-PSY Study is supported by peer reviewed grants of the French Ministry of Health (PHRCl-19-063) and the Fondation de l'Avenir (AP-RM-19-011).

Competing interests FH reports receipt of personal fees or non-financial support not directly related to this study from Janssen, Lundbeck and Otsuka. EF reports receipt of personal fees or non-financial support not directly related to this study from Janssen, Lundbeck, Otsuka, Recordatti, Sanofi, AbbVie, MSD. JH reports receipt of personal fees or non-financial support not directly related to this study from Janssen. SE0, AP, DF, CC, LM, ED and FS report no financial relationships with commercial interests.

Patient consent for publication Not required.

Provenance and peer review Not commissioned; externally peer reviewed.

Supplemental material This content has been supplied by the author(s). It has not been vetted by BMJ Publishing Group Limited (BMJ) and may not have been peer-reviewed. Any opinions or recommendations discussed are solely those of the author(s) and are not endorsed by BMJ. BMJ disclaims all liability and responsibility arising from any reliance placed on the content. Where the content includes any translated material, BMJ does not warrant the accuracy and reliability of the translations (including but not limited to local regulations, clinical guidelines, terminology, drug names and drug dosages), and is not responsible for any error and/or omissions arising from translation and adaptation or otherwise.

Open access This is an open access article distributed in accordance with the Creative Commons Attribution Non Commercial (CC BY-NC 4.0) license, which permits others to distribute, remix, adapt, build upon this work non-commercially, and license their derivative works on different terms, provided the original work is properly cited, appropriate credit is given, any changes made indicated, and the use is non-commercial. See: http://creativecommons.org/licenses/by-nc/4.0/.

ORCID iD

Frédéric Haesebaert http://orcid.org/0000-0002-6813-9012

\section{REFERENCES}

1 Kirkbride JB, Hameed Y, Ankireddypalli G, et al. The Epidemiology of First-Episode Psychosis in Early Intervention in Psychosis Services: Findings From the Social Epidemiology of Psychoses in East Anglia [SEPEA] Study. Am J Psychiatry 2017;174:143-53.

2 Perälä J, Suvisaari J, Saarni SI, et al. Lifetime prevalence of psychotic and bipolar I disorders in a general population. Arch Gen Psychiatry 2007;64:19-28.

3 Pompili M, Serafini G, Innamorati M, et al. Suicide risk in first episode psychosis: a selective review of the current literature. Schizophr Res 2011;129:1-11.

4 Birchwood M, Todd P, Jackson C. Early intervention in psychosis. The critical period hypothesis. Br J Psychiatry 1998;172:53-9.

5 Penttilä M, Jääskeläinen E, Hirvonen N, et al. Duration of untreated psychosis as predictor of long-term outcome in schizophrenia: systematic review and meta-analysis. Br J Psychiatry 2014;205:88-94.

6 Nordentoft M, Madsen T, Fedyszyn I. Suicidal behavior and mortality in first-episode psychosis. J Nerv Ment Dis 2015;203:387-92.

7 Shiers D, Jones PB, Field S. Early intervention in psychosis: keeping the body in mind. Br J Gen Pract 2009;59:395.

8 Berk LE. Emotional and social development in early adulthood. In: Development through the lifespan, 2018.

9 McGorry PD. Early intervention in psychosis: obvious, effective, overdue. J Nerv Ment Dis 2015;203:310-8.

10 Correll CU, Galling B, Pawar A, et al. Comparison of early intervention services vs treatment as usual for early-phase psychosis: a systematic review, meta-analysis, and meta-regression. JAMA Psychiatry 2018;75:555-65.

11 McGorry PD, Jackson HJ. The recognition and management of early psychosis : a preventive approach. Cambridge; New York: Cambridge University Press, 1999.

12 Holloway F, Oliver N, Collins E, et al. Case management: a critical review of the outcome literature. Eur Psychiatry 1995;10:113-28.

13 Early Psychosis Guidelines Writing Group and EPPIC National Support Program. Australian clinical guidelines for early psychosis. 2nd edn. Orygen, Melbourne: The National Centre of Excellence in Youth Mental Health, 2016.

14 Lal S, Malla A. Service engagement in First-Episode psychosis: current issues and future directions. Can J Psychiatry 2015;60:341-5.

15 Alvarez-Jimenez M, Priede A, Hetrick SE, et al. Risk factors for relapse following treatment for first episode psychosis: a systematic review and meta-analysis of longitudinal studies. Schizophr Res 2012;139:116-28.

16 Di Capite S, Upthegrove R, Mallikarjun P. The relapse rate and predictors of relapse in patients with first-episode psychosis following discontinuation of antipsychotic medication. Early Interv Psychiatry 2018;12:893-9.

17 Dixon LB, Holoshitz Y, Nossel I. Treatment engagement of individuals experiencing mental illness: review and update. World Psychiatry 2016;15:13-20.

18 Smith TE, Easter A, Pollock M, et al. Disengagement from care: perspectives of individuals with serious mental illness and of service providers. Psychiatr Serv 2013;64:770-5.

19 Mcdaid D, Park A-L, lemmi V, et al. Growth in the use of early intervention for psychosis services: an opportunity to promote recovery amid concerns on health care sustainability, 2016.

20 Schürhoff $F$, Fond $G$, Berna $F$, et al. A national network of schizophrenia expert centres: an innovative tool to bridge the research-practice gap. Eur Psychiatry 2015;30:728-35.

21 Courouve L, Duburcq A, Gozlan G, et al. Mapping of early intervention programs for psychosis in France in 2018. Eur J Public Health 2019;29.

22 Lal S, Malla A. Service engagement in first-episode psychosis: current issues and future directions. Can J Psychiatry 2015;60:341-5.

23 Torous J, Woodyatt J, Keshavan M, et al. A new hope for early psychosis care: the evolving landscape of digital care tools. $\mathrm{Br} \mathrm{J}$ Psychiatry 2019;214:269-72.

24 Firth J, Cotter J, Torous J, et al. And endorsement of «mHealth " among people with psychosis: a meta-analysis of cross-sectional studies. Schizophr Bull. mars 2016;42:448-55.

25 Niendam TA, Tully LM, losif A-M, et al. Enhancing early psychosis treatment using smartphone technology: a longitudinal feasibility and validity study. J Psychiatr Res 2018;96:239-46.

26 Hardy A, Wojdecka A, West J, et al. How inclusive, User-Centered design research can improve psychological therapies for psychosis: development of SlowMo. JMIR Ment Health 2018;5:e11222.

27 Ramsay CE, Broussard B, Goulding SM, et al. Life and treatment goals of individuals hospitalized for first-episode nonaffective psychosis. Psychiatry Res 2011;189:344-8.

28 Whitley R, Drake RE. Recovery: a dimensional approach. Psychiatr Serv 2010;61:1248-50.

29 Pomini V, Bonsack C. Chapitre 6 - Modèles théoriques du rétablissement. In: Franck N, ed. Traité de Réhabilitation Psychosociale [online. Paris: Elsevier Masson, 2018: 55-63. http:// www.sciencedirect.com/science/article/pii/B9782294759154000062

30 Zhou L, DeAlmeida D, Parmanto B. Applying a user-centered approach to building a mobile personal health record APP: development and usability study. JMIR Mhealth Uhealth 2019;7:e13194.

31 Torous J, Wisniewski H, Liu G, et al. Mental health mobile phone APP usage, concerns, and benefits among psychiatric outpatients: comparative survey study. JMIR Ment Health 2018;5:e11715.

32 McGorry PD, Edwards J, Mihalopoulos C, et al. EPPIC: an evolving system of early detection and optimal management. Schizophr Bull 1996;22:305-26.

33 Morosini PL, Magliano L, Brambilla L, et al. Development, reliability and acceptability of a new version of the DSM-IV social and occupational functioning assessment scale (SOFAS) to assess routine social functioning. Acta Psychiatr Scand 2000;101:323-9.

34 Tait L, Birchwood M, Trower P. A new scale (Ses) to measure engagement with community mental health services. $J$ Ment Health 2002;11:191-8.

35 Horvath AO, Greenberg LS. Development and validation of the working alliance inventory. J Couns Psychol 1989;36:223-33.

36 Thompson K, Kulkarni J, Sergejew AA. Reliability and validity of a new medication adherence rating scale (MARS) for the psychoses. Schizophr Res 2000;42:241-7. 
37 Kay SR, Fiszbein A, Opler LA. The positive and negative syndrome scale (PANSS) for schizophrenia. Schizophr Bull 1987;13:261-76.

38 Andresen R, Caputi P, Oades L. Stages of recovery instrument: development of a measure of recovery from serious mental illness. Aust N Z J Psychiatry 2006;40:972-80.

39 Boyer L, Simeoni M-C, Loundou A, et al. The development of the S-QoL 18: a shortened quality of life questionnaire for patients with schizophrenia. Schizophr Res 2010;121:241-50.

40 Chang WC, Kwong VWY, Lau ESK, et al. Sustainability of treatment effect of a 3-year early intervention programme for first-episode psychosis. Br J Psychiatry 2017;211:37-44.

41 Fleiss JL, Tytun A, Ury HK. A simple approximation for calculating sample sizes for comparing independent proportions. Biometrics 1980;36:343-6.

42 R Foundation for Statistical Computing. Core team R. R: a language and environment for statistical computing. Vienna, 2020.

43 Nevarez-Flores AG, Sanderson K, Breslin M, et al. Systematic review of global functioning and quality of life in people with psychotic disorders. Epidemiol Psychiatr Sci 2019;28:31-44.
44 Blanc O, Delgado A, Lancon C, et al. Psychometric properties of the French version of the personal and social performance scale (PSP) among individuals with schizophrenia. Schizophrenia Research 2010:117:285-6.

45 Martinuzzi E, Barbosa S, Daoudlarian D. Bel Haj Ali W, Gilet C, Fillatre $L$, et al. stratification and prediction of remission in firstepisode psychosis patients: the optimise cohort study. Transl Psychiatry 2019;9:20.

46 Albert N, Melau M, Jensen $\mathrm{H}$, et al. Five years of specialised early intervention versus two years of specialised early intervention followed by three years of standard treatment for patients with a first episode psychosis: randomised, superiority, parallel group trial in Denmark (OPUS II). BMJ. 12 janv 2017;356:i6681.

47 Alvarez-Jimenez M, Koval P, Schmaal L, et al. The Horyzons project: a randomized controlled trial of a novel online social therapy to maintain treatment effects from specialist first-episode psychosis services. World Psychiatry 2021;20:233-43.

48 Faay MDM, van Baal GCM, Arango C, et al. Hostility and aggressive behaviour in first episode psychosis: results from the optimise trial. Schizophr Res 2020;223:271-8. 